\title{
Characteristics and Management of Ruptured Aneurysms Originating from the Penetrating Artery of the Distal Middle Cerebral Artery
}

\author{
Yu IIDA, ${ }^{1}$ Kentaro MORI, ${ }^{1}$ Yosuke KAWAHARA, ${ }^{1}$ Issei FUKUI, ${ }^{1}$ Katsuya ABE, ${ }^{2}$ \\ Mutsuki TAKedA, ${ }^{2}$ Tatsu NAKAnO, ${ }^{2}$ Kunio YanAgIMOTO, ${ }^{3}$ \\ and Motohiro NOMURA ${ }^{1}$
}

${ }^{1}$ Department of Neurosurgery, Yokohama Sakae Kyosai Hospital, Yokohama, Kanagawa, Japan ${ }^{2}$ Department of Neurology, Yokohama Sakae Kyosai Hospital, Yokohama, Kanagawa, Japan ${ }^{3}$ Department of Pathology, Yokohama Sakae Kyosai Hospital, Yokohama, Kanagawa, Japan

\begin{abstract}
We report a rare case of an aneurysm originating from the penetrating artery of the distal middle cerebral artery (MCA). A 76-year-old man without a notable past history presented with sudden-onset severe headache, left hemiparesis, and a decreased level of consciousness. Computed tomography (CT) revealed subarachnoid hemorrhage (SAH) with intracerebral hemorrhage (ICH) in the right temporal lobe extending into the ventricle. Contrast-enhanced CT (CE-CT) demonstrated a focus of contrast enhancement (CE) adjacent to the hematoma in the right frontal lobe. An aneurysm fed by a penetrating artery branching off from the right distal MCA was found on angiography. The patient underwent emergency resection of the aneurysm and hematoma evacuation. Histological analysis revealed that arterial dissection may be an associated factor in the pathogenesis of this peripheral aneurysm formation. A focus of CE within or adjacent to the hematoma may be useful for diagnosing this peripheral aneurysm. ICH can result in a life-threatening situation. Therefore, microsurgery may be the first treatment choice for aneurysms in this location.
\end{abstract}

Keywords: distal middle cerebral artery, penetrating artery, intracerebral hemorrhage, aneurysm, dissection

\section{Introduction}

Distal middle cerebral artery (MCA) aneurysms are rare, accounting for $2-6 \%$ of all MCA aneurysms. ${ }^{1-3)}$ Most of them are located in the main trunk of each MCA segment. On the other hand, aneurysms originating from the penetrating arteries of the distal MCA are markedly rare. To our knowledge, only three cases including ours have been reported in the English literature. ${ }^{4,5)}$ The nature and management of this rare aneurysm have not been fully clarified. Recently, we treated a patient with an aneurysm originating from the penetrating artery of the distal MCA. In this report, we describe the treatment of

Received January 8, 2020; Accepted April 9, 2020

Copyright $\subseteq 2021$ by The Japan Neurosurgical Society This work is licensed under a Creative Commons AttributionNonCommercial-NoDerivatives International License. the patient, and discuss the nature and management of this rare aneurysm with a literature review.

\section{Case Presentation}

A 76-year-old man was brought to our hospital with sudden-onset severe headache, left hemiparesis, and consciousness disturbance. The medical history of the patient was unremarkable. His blood pressure was 179/98 $\mathrm{mmHg}$, heart rate 75 beats/min, and temperature $36.4^{\circ} \mathrm{C}$. Laboratory analysis showed normal white blood cell count and C-reactive protein was $<0.1 \mathrm{mg} / \mathrm{dL}$. No preceding head trauma or infection was noted. Echocardiography showed no abnormal findings. Computed tomography (CT) revealed subarachnoid hemorrhage (SAH) and intracerebral hemorrhage (ICH) in the right temporal lobe with ventricular rupture (Fig. 1A). Contrastenhanced CT (CE-CT) demonstrated a focus of contrast enhancement (CE) adjacent to the hematoma in the 

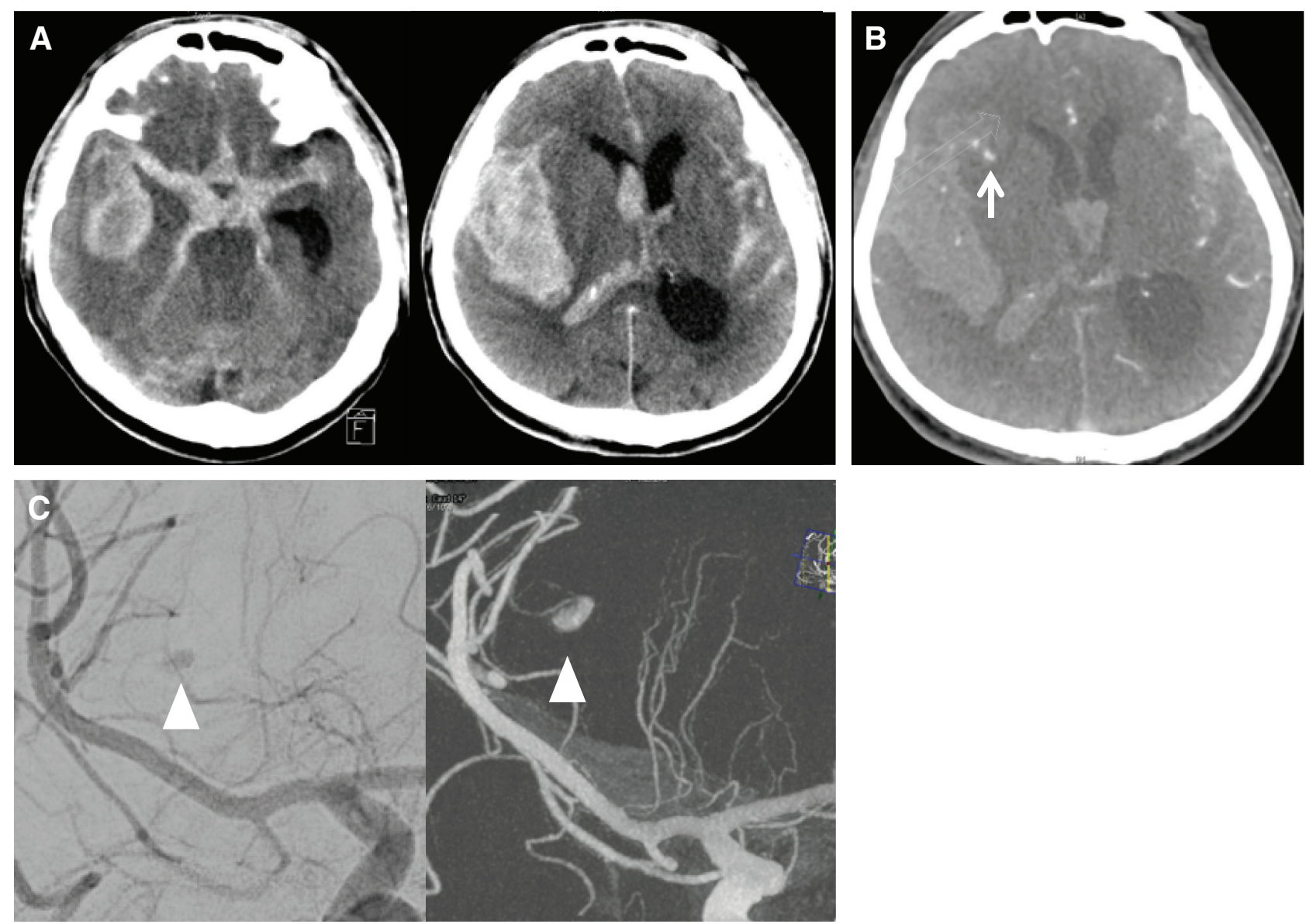

Fig. 1 (A) CT showing SAH and ICH in the right temporal lobe extending into the ventricle. (B) Contrastenhanced CT demonstrating a focus of CE adjacent to the hematoma in the right frontal lobe (arrow). (C) Angiography showing an aneurysm fed by a penetrating artery branching off from the right distal MCA (arrowheads). Left panel: conventional image, right: maximum intensity projection image. CE: contrast enhancement, CT: computed tomography, ICH: intracerebral hemorrhage, MCA: middle cerebral artery, SAH: subarachnoid hemorrhage.
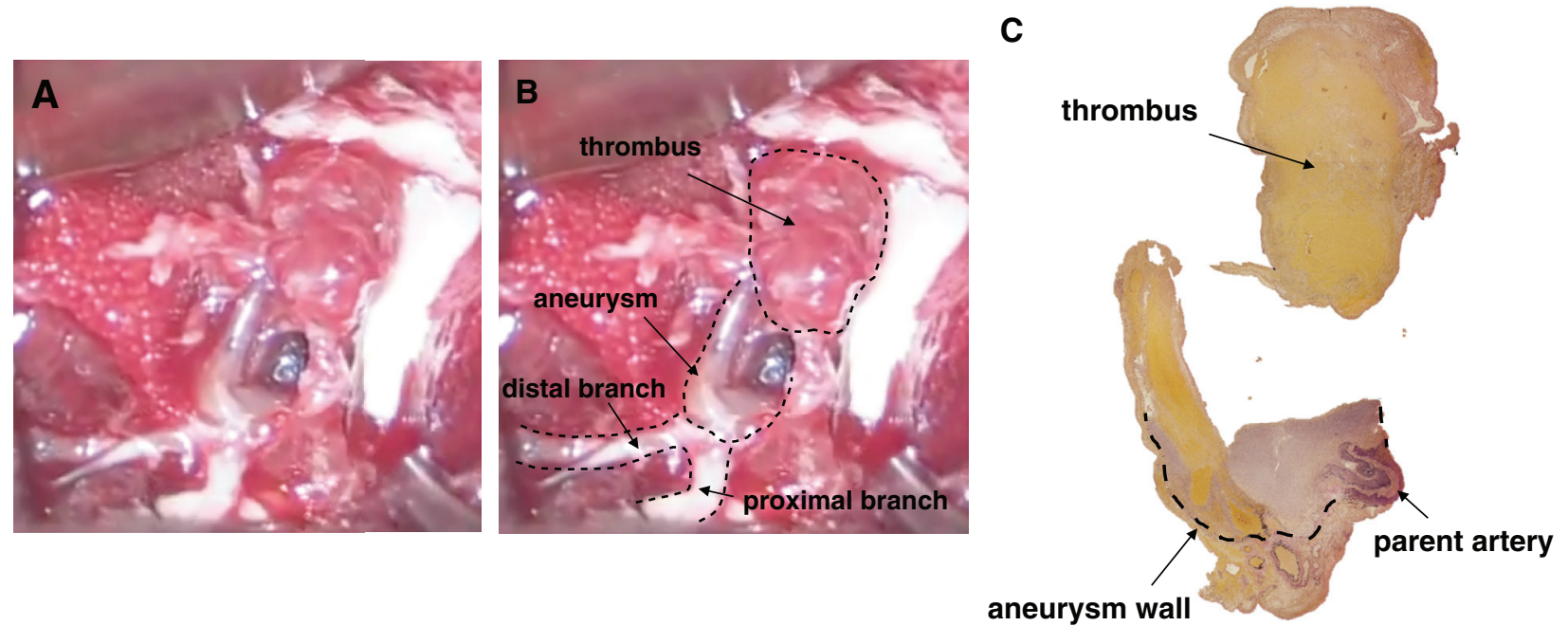

Fig. 2 An intraoperative photograph (A) and the illustrative drawing (B) demonstrating the aneurysm originating from the M2 penetrating artery. Photomicrograph of histopathological examination corresponding to the intraoperative photograph (C) showing disruption of the aneurysm wall and the thrombus. 

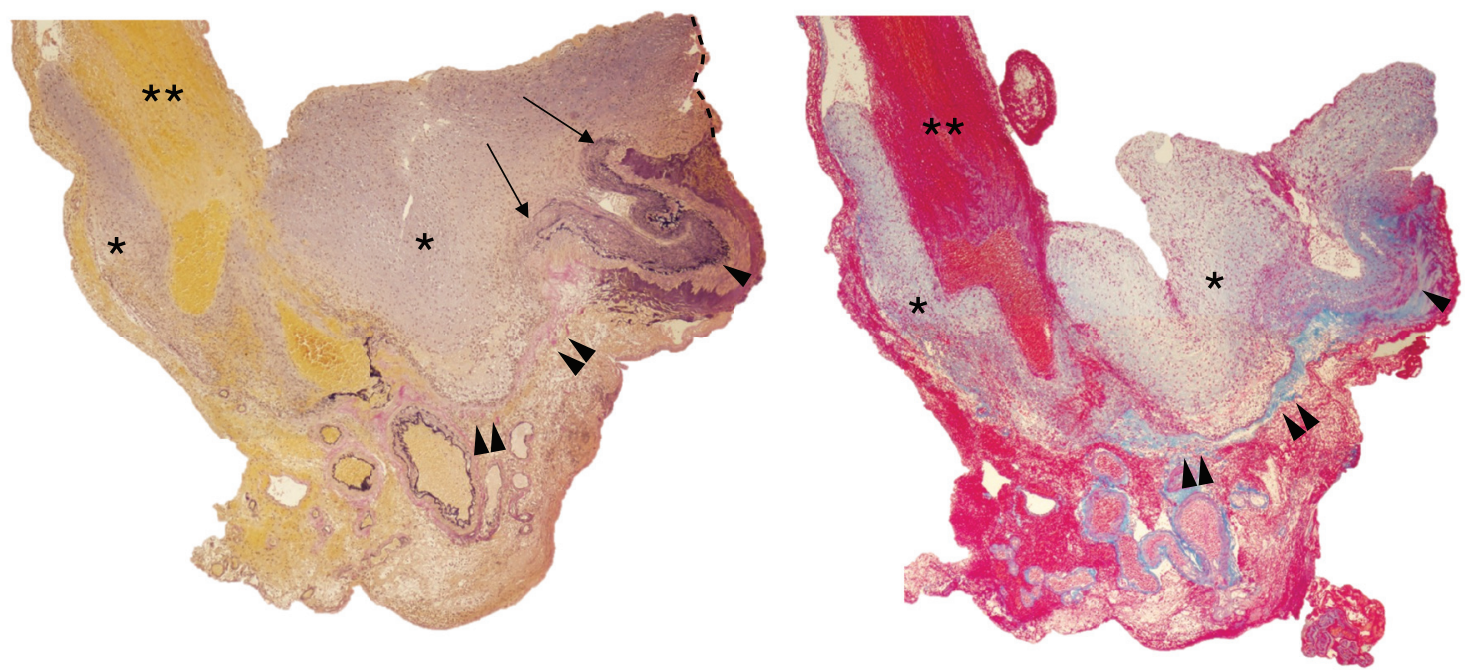

Fig. 3 Close-up view of aneurysm wall of Fig. 2C showing disrupted ends of the IEL and media (arrows) of the parent artery (arrowhead). The aneurysm wall is lack of the IEL and covered by the neointima (asterisks) with the thin adventitia (double arrowheads). The neointima attached on the fresh thrombus (double asterisks) is relatively thin. Left: Elastica van Gieson staining, right: Masson Trichrome staining, IEL: internal elastic lamina.

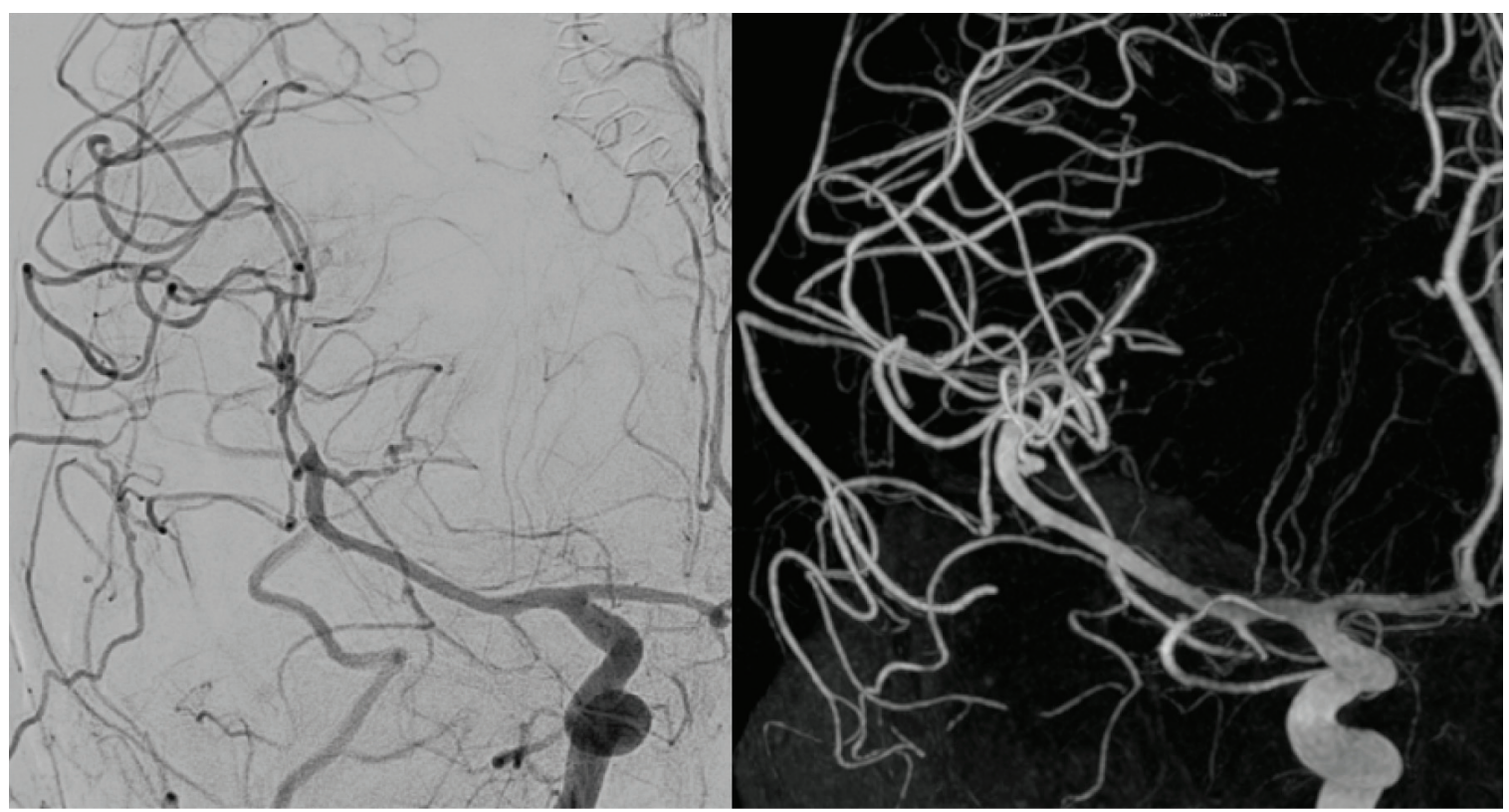

Fig. 4 Postoperative angiography showing disappearance of the aneurysm. Left panel: conventional image, right: maximum intensity projection image.

right frontal lobe (Fig. 1B). An aneurysm with a diameter of $4 \mathrm{~mm}$ was found on angiography. The aneurysm was fed by a penetrating artery branching off from the right distal MCA (M2 segment) (Fig. 1C). In addition, no bacterial organisms were detected by blood culture. We diagnosed the hemorrhage as being due to rupture of the aneurysm originating from the penetrating artery of the distal MCA. Emergency right fronto-temporal craniotomy was performed. The Sylvian fissure was opened, and the right MCA and its penetrating artery were exposed. The hematoma in the right temporal lobe was evacuated. A saccular aneurysm originating from the M2 penetrating artery was embedded in the hematoma in the right frontal lobe (Fig. 2). The aneurysm countenance was the same as common aneurysms. A thrombus was attached to the aneurysm dome. Examination by Doppler ultrasound sonography revealed no blood flow in the penetrating artery. Therefore, the aneurysm including the parent 
artery was resected. Bleeding from the parent artery was not observed after resection. Histological analysis revealed disruption of the internal elastic lamina (IEL) and media of the parent artery. The aneurysm wall was lack of IEL, and covered irregular structure of the neointima with the elongated adventitia. Fresh thrombus was also observed within the aneurysm. No infecting pathogens, such as fungus, bacteria, or phagocytosis by immune cells, were detected around the wall (Fig. 3). Based on these findings, the dissecting aneurysm originating from the penetrating artery of the distal MCA was suspected. Postoperatively, the patient had no additional neurological deficit. Postoperative angiography demonstrated disappearance of the aneurysm (Fig. 4). On the 28th day, left ventriculo-peritoneal shunting was performed. He was transferred to another hospital for rehabilitation on the 67th day.

\section{Discussion}

Since Poppen ${ }^{6}$ first reported cases of distal MCA aneurysms in 1951, several case series of ruptured distal MCA aneurysms have been reported. Distal MCA aneurysms originate along the main trunk of each segment, and the M2 segment is the most commonly affected. ${ }^{1)}$ On the other hand, several reports describing aneurysms originating from small perforating or penetrating branches, such as lenticulostriate artery (LSA), ${ }^{7)}$ anterior choroidal, ${ }^{8,9)}$ thalamostriate, ${ }^{10)}$ and distal basilar arteries, ${ }^{11)}$ have been published. As for a penetrating artery of the distal MCA, aneurysms originating from this artery are rare. To our knowledge, only three cases including ours have been reported (Table 1).,5) Peripherally located aneurysms are commonly associated with infection, tumor embolus, moyamoya disease, arteriovenous malformation, or trauma. ${ }^{7}$ However, there was no aforementioned cause for aneurysms originating from the penetrating artery of the distal MCA. Patient ages varied from 20 to 76 (average 44.3) years, and two patients were relatively young compared with those with common saccular aneurysms. One previous case was diagnosed as the dissecting aneurysm originating from penetrating branch of the distal MCA on the basis of the histological findings. ${ }^{5)}$ Interestingly, in 263 spontaneous arterial dissections at the Mayo Clinic, intraarterial dissections were relatively more common in children and adolescents than in adults. ${ }^{12)}$ In our case, arterial dissection of penetrating artery was also observed. This suggests that this type of aneurysm is associated with factors other than atherosclerosis. Mizutani et al. ${ }^{13)}$ reported arterial dissections of penetrating cerebral arteries causing cerebral hemorrhage. They performed pathological studies in

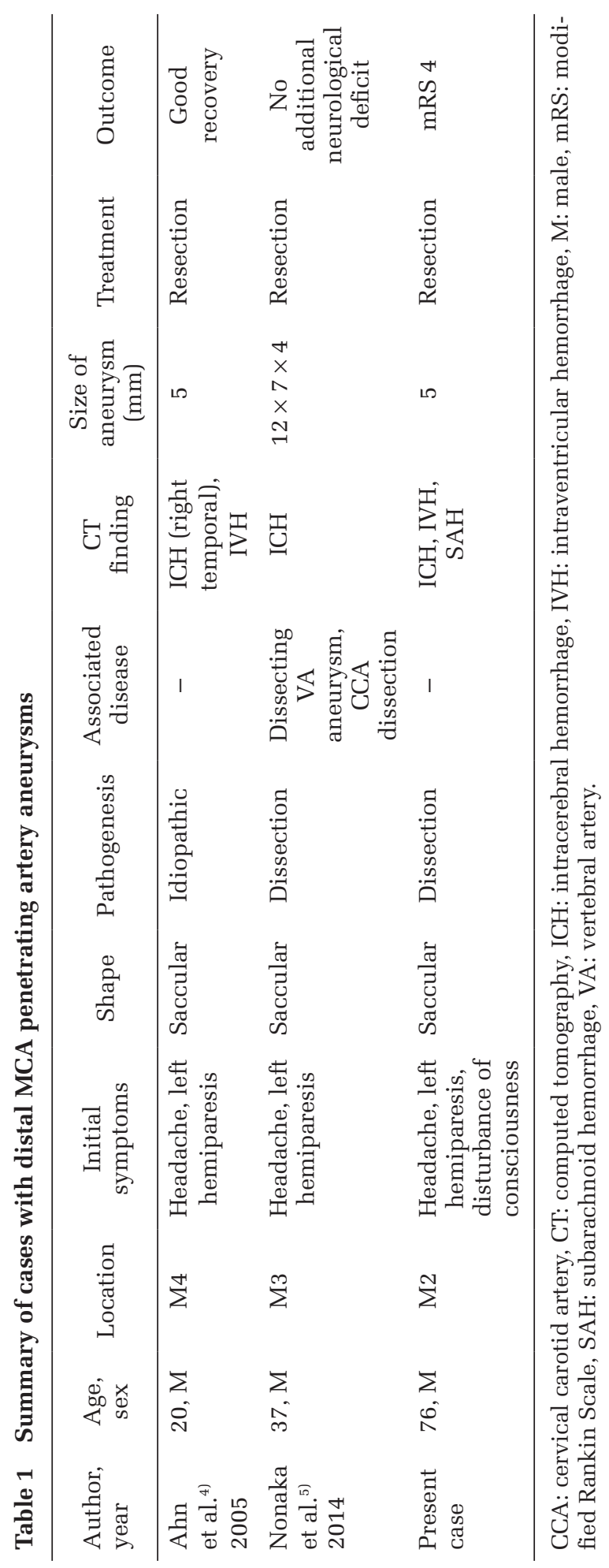

NMC Case Report Journal 8, February, 2021 

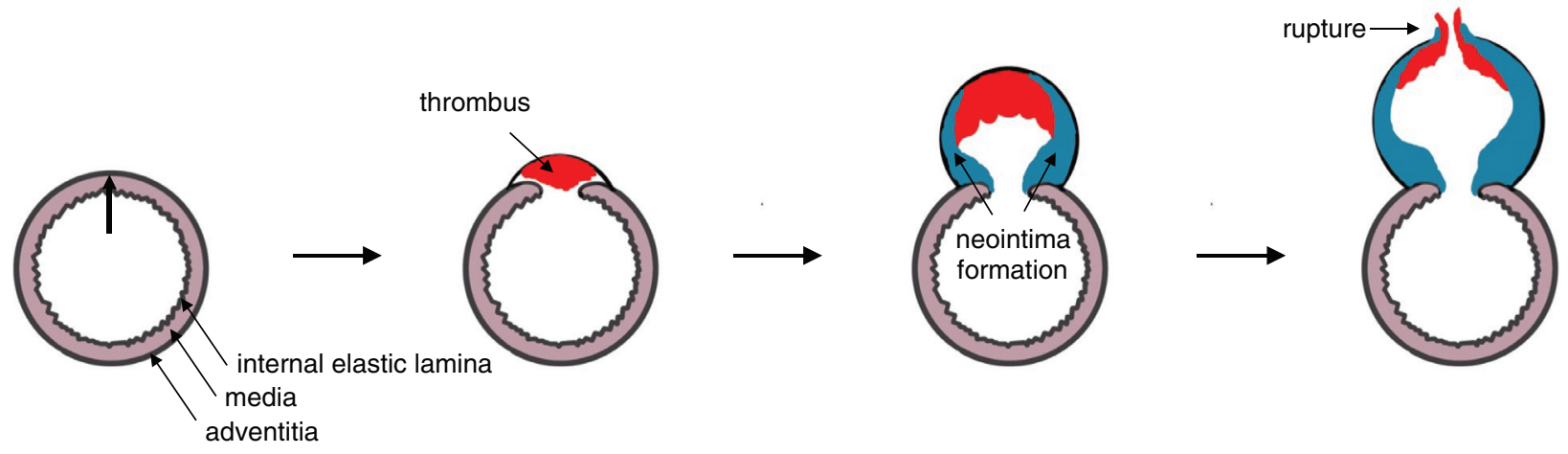

Fig. 5 Illustration of the aneurysm formation from arterial dissection to aneurysm rupture of the penetrating artery.

surgical specimens of LSA that had been confirmed during microsurgery to be the cause of the putamen hemorrhage. Fifteen of these arteries were verified as the pathological causes of hemorrhage, and included six arterial dissections, and nine arterial rupture. They assumed that arterial dissections of the penetrating cerebral arteries may be more common causes of cerebral hemorrhage. Mizutani et al. ${ }^{14)}$ also reported healing process for cerebral dissecting aneurysms. The healing process of the injured arterial wall occurred with neointimal proliferation, which appears after 1 week. At 3 months, neointima formation ceases. The neointima is consisted primarily of newly synthesized smooth muscle cells and an extracellular matrix with collagen fibers, elastic fibers, proteoglycans, and other components. Vascular wall repair via neointima formation occurred from the distal ends of the media toward the ruptured portion. Wall repair is completed after the neointima covers the entire area of the arterial wall. When the neointima did not reach the ruptured portion of the aneurysm, pathological confirmation of safety from the risk of rebleeding was not obtained. In the present case, we speculate that disruptions of the IEL and media were occurred, but not developed into cerebral hemorrhage. After disruptions of the IEL and media, neointima structure was gradually developed from the disrupted ends of the IEL and media, while aneurysmal formation was developed. However, aneurysm rupture was occurred during healing process (Fig. 5). In the present histological analysis, the thickness of the neointima was observed at the disrupted ends of the IEL and media. On the other hand, the neointima attached on the fresh thrombus was relatively thin. These findings suggested that the neointima did not reach the ruptured portion of the aneurysm, or it was not strong enough to sustain the hemodynamic stress. These aforementioned findings suggested that arterial dissection may be an associated factor in the pathogenesis of this peripheral aneurysm.
The incidence of ICH in cases of ruptured MCA aneurysms was reported to be as high as $44 \% .^{6,15)}$ Of the previous studies of ruptured distal MCA aneurysms, a total of 36 of 60 patients had associated ICH. ${ }^{16)}$ Regarding aneurysms in the penetrating artery of the distal MCA, all three cases presented with ICH, and only our case had accompanying SAH and ICH (Table 1). One previous case was initially treated under the diagnosis of subcortical hemorrhage of unknown etiology, but distal MCA aneurysm was later identified on angiography. ${ }^{5}$ In a case of only ICH, it is difficult to suspect an aneurysm. In our case, the initial radiological examination of CE-CT revealed the focus of $\mathrm{CE}$ adjacent to the hematoma in the right frontal lobe. Therefore, we additionally performed angiography to evaluating this focus. As a result, the penetrating artery aneurysm of the distal MCA was identified. Although angiography may not be commonly performed for simple ICH, there is a possibility of a penetrating artery aneurysm in cases with a spotty CE lesion adjacent to the hematoma. Tan et al. ${ }^{17)}$ reported the spot sign in a case of LSA aneurysm on CE-CT or CE-magnetic resonance imaging, and concluded that the spot sign may be extravasation of contrast medium into the hematoma. Thus, an aneurysm originating from the penetrating artery of the distal MCA commonly presents with ICH and is not always accompanying SAH. Therefore, the focus of CE within or adjacent to the hematoma on CE-CT may be useful for diagnosing this aneurysm, even if the hematoma is normal.

All three patients underwent surgical treatment, and trapping and aneurysm resection were performed. All patients had no additional neurological deficit after the operation. Recently, endovascular treatment has been widely used to treat distal cerebral aneurysms with good outcomes. ${ }^{18-20)}$ The endovascular approach may be more advantageous than microsurgery for aneurysms located in the main trunk of each MCA segment because of the accessibility to the lesion. 
However, perforating or penetrating arteries are thin, and insertion and advancement of a microcatheter are difficult. Therefore, endovascular embolization of the aneurysm at this location may be challenging. In addition, Joo et al. ${ }^{2)}$ reported that patients with distal MCA aneurysms causing ICH had unfavorable outcomes. As for patients with ruptured aneurysms in the penetrating artery of the distal MCA, all had accompanying ICH. As ICH can result in a life-threatening situation, early surgical removal of the hematoma and aneurysm occlusion are essential.

\section{Conclusion}

Aneurysms originating from the penetrating artery of the distal MCA are markedly rare. Arterial dissection may be associated with this peripheral aneurysm formation. Such aneurysms are commonly accompanied by ICH, but not SAH. A focus of CE within or adjacent to the hematoma may be useful for diagnosing these peripheral aneurysms. Microsurgical trapping of an aneurysm and removal of the hematoma may be the first treatment choice because controlling brain swelling and preventing re-bleeding are important.

\section{Conflicts of Interest Disclosure}

The authors have no personal financial or institutional interests in any of the drugs, materials, or devices in the article.

\section{References}

1) Horiuchi T, Tanaka Y, Takasawa H, Murata T, Yako T, Hongo K: Ruptured distal middle cerebral artery aneurysm. J Neurosurg 100: 384-388, 2004

2) Joo SP, Kim TS, Choi JW, et al.: Characteristics and management of ruptured distal middle cerebral artery aneurysms. Acta Neurochir (Wien) 149: 661-667, 2007

3) Rinne J, Hernesniemi J, Niskanen M, Vapalahti M: Analysis of 561 patients with 690 middle cerebral artery aneurysms: anatomic and clinical features as correlated to management outcome. Neurosurgery 38: 2-11, 1996

4) Ahn JY, Han IB, Joo JY: Aneurysm in the penetrating artery of the distal middle cerebral artery presenting as intracerebral haemorrhage. Acta Neurochir (Wien) 147: 1287-1290, discussion 1290, 2005

5) Nonaka S, Tsutsumi S, Suzuki T, Izumi H, Yasumoto Y: Distal middle cerebral artery aneurysm manifesting as subcortical hemorrhage. Neurosurg Emerg 19: 226-229, 2014

6) Poppen JL: Specific treatment of intracranial aneurysms; experiences with 143 surgically treated patients. J Neurosurg 8: 75-102, 1951
7) Nomura M, Baba E, Shirokane K, Tsuchiya A: Aneurysm of lenticulostriate artery in a patient presenting with hemorrhage in the caudate nucleus and lateral ventricle-delayed appearance and spontaneous resolution. Surg Neurol Int 9: 192, 2018

8) Strully KJ: Successful removal of intraventricular aneurysm of the choroidal artery. J Neurosurg 12: 317-321, 1955

9) Tan CH, Lai LT, Chandra RV: Ruptured distal anterior choroidal artery aneurysm presenting as isolated intraventricular hemorrhage: case report and comprehensive review of the literature. Asian J Neurosurg 13: 487-491, 2018

10) Bergsneider M, Frazee JG, DeSalles AA: Thalamostriate artery aneurysm within the third ventricle. Case report. J Neurosurg 81: 463-465, 1994

11) Ghogawala Z, Shumacher JM, Ogilvy CS: Distal basilar perforator artery aneurysm: case report. Neurosurgery 39: 393-396, 1996

12) Schievink WI, Mokri B, Piepgras DG: Spontaneous dissections of cervicocephalic arteries in childhood and adolescence. Neurology 44: 1607-1612, 1994

13) Mizutani T, Kojima H, Miki Y: Arterial dissections of penetrating cerebral arteries causing hypertension-induced cerebral hemorrhage. J Neurosurg 93: 859-862, 2000

14) Mizutani T, Kojima H, Asamoto S: Healing process for cerebral dissecting aneurysms presenting with subarachnoid hemorrhage. Neurosurgery 54: 342347; discussion 347-348, 2004

15) Teasdale GM, Drake CG, Hunt W, et al.: A universal subarachnoid hemorrhage scale: report of a committee of the World Federation of Neurosurgical Societies. J Neurol Neurosurg Psychiatry 51: 1457, 1988

16) Tsutsumi K, Horiuchi T, Nagm A, Toba Y, Hongo K: Clinical characteristics of ruptured distal middle cerebral artery aneurysms: review of the literature. J Clin Neurosci 40: 14-17, 2017

17) Tan LA, Kasliwal MK, Johnson AK, Lopes DK: The "Spot Sign" secondary to a ruptured lenticulostriate artery aneurysm. Clin Imaging 38: 508-509, 2014

18) Baltacioğlu F, Cekirge S, Saatci I, et al.: Distal middle cerebral artery aneurysms. Endovascular treatment results with literature review. Interv Neuroradiol 8: 399-407, 2002

19) Darkhabani ZM, Lazzaro MA, Zaidat OO: Pericallosal artery aneurysm treatment using Y-configuration stent-assisted coil embolization: a report of four cases. J Neurointerv Surg 4: 459-462, 2012

20) Peluso JP, van Rooij WJ, Sluzewski M, Beute GN: Distal aneurysms of cerebellar arteries: incidence, clinical presentation, and outcome of endovascular parent vessel occlusion. AJNR Am J Neuroradiol 28: 1573-1578, 2007

Corresponding author: Yu Iida, MD

Department of Neurosurgery, Yokohama Sakae Kyosai Hospital, 132 Katsuracho, Sakae-ku, Yokohama 247-8581, Japan e-mail: yuiida1204@gmail.com 